\title{
Short-distance constraints in hadronic-light-by-light for the muon $g-2$
}

\author{
Johan Bijnens, ${ }^{a}{ }^{*}$ Nils Hermansson-Truedsson ${ }^{b}$ and Antonio Rodríguez Sánchez ${ }^{c}$ \\ ${ }^{a}$ Department of Astronomy and Theoretical Physics, Lund University, \\ Box 43, SE221-00 Lund, Sweden \\ ${ }^{b}$ Albert Einstein Center for Fundamental Physics, Institute for Theoretical Physics, Universität Bern, \\ Sidlerstrasse 5, CH-3012 Bern, Switzerland \\ ${ }^{c}$ Université Paris-Saclay, CNRS/IN2P3, IJCLab, \\ 91405 Orsay, France \\ E-mail: johan.bijnens@thep.lu.se, nils@itp.unibe.ch, \\ arodriguez@ijclab.in2p3.fr
}

In this talk recent progress in studying the short-distance properties of the hadronic light-by-light contribution to the muon $g-2$ is described. The intermediate and short-distance part is a major contributor to the error of the theoretical prediction as described in the recent white paper [1]. We have shown that the massless quark-loop is the first term in a systematic expansion at shortdistances, a result already used in the white paper. Newer results conclude that both nonperturbative and perturbative corrections are under control. The talk describes these developments and how they fit in the total theoretical prediction for the muon $g-2$.

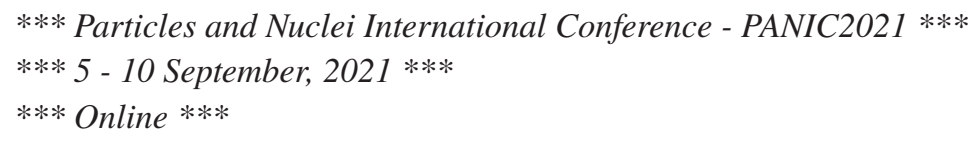

\footnotetext{
${ }^{*}$ Speaker
} 


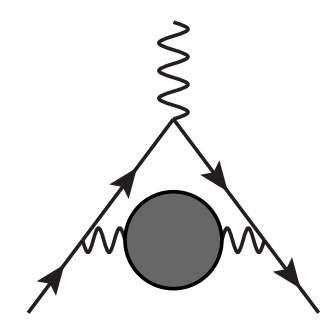

LO-HVP

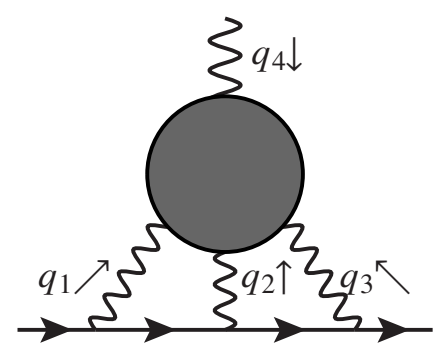

HLbL

Figure 1: The LO-HVP and HLbL contributions shown schematically. The arrowed line is the muon, wiggly lines indicate photons and the blobs indicate hadronic contributions.

\begin{tabular}{lrlr}
\hline \multicolumn{2}{c}{ Long distance } & \multicolumn{2}{c}{ Short and medium distance } \\
\hline$\pi^{0}$ (and $\eta, \eta^{\prime}$ ) pole & $93.8(4.0) 10^{-11}$ & Charm (beauty, top) loop & $3(1) 10^{-11}$ \\
Pion and kaon box (pure) & $-16.4(2) 10^{-11}$ & Axial vector & $6(6) 10^{-11}$ \\
$\pi \pi$-rescattering & $-8(1) 10^{-11}$ & Short-distance & $15(10) 10^{-11}$ \\
\hline
\end{tabular}

Table 1: The main contributions to the phenomenological evaluation of HLbL as estimated in [1]. Scalars below $1 \mathrm{GeV}$ are included in $\pi \pi$-rescattering. Scalars above $1 \mathrm{GeV}$ are small.

\section{Introduction}

There is a long standing difference between the Standard Model (SM) prediction [1] for the muon anomalous magnetic moment $a_{\mu}=(g-2) / 2$ and the experimental measurement [2] at BNL, recently confirmed by the experiment at FNAL $[3,4]$. The theoretical prediction, $a_{\mu}^{S M}$, and experimental average, $a_{\mu}^{\text {exp }}$, give a difference $\Delta a_{\mu}=251(59) \times 10^{-11}$. corresponding to $4.2 \sigma$.

The theoretical error is dominated by two hadronic contributions, the lowest order hadronic vacuum polarization (LO-HVP) and the hadronic light-by-light contribution (HLbL), shown schematically in Fig. 1. The higher order hadronic, electroweak and QED contributions do not significantly contribute to the theoretical error. The error at the moment is dominated by the LO-HVP but improvements on both the dispersive and lattice calculations are expected.

The HLbL contribution and its error as estimated in the white paper [1] are $a_{\mu}^{H L b L}=$ 92(18) $10^{-11}$. Improvements since then include an improved lattice QCD calculation [5] compatible with the earlier lattice results [6] and [1]. The main problem with the HLbL calculation is that, as shown in the right figure of Fig. 1, its evaluation always has one very low momentum, $q_{4}$, i.e. the external magnetic field, and momenta $q_{1}, q_{2}, q_{3}$ which span the entire range, both low and high. For a long time this only allowed for model calculations, see e.g. [7-12]. More recently $[13,14]$ produced a dispersive method allowing better control of the long-distance parts. Tab. 1 summarizes the phenomenological parts of the HLbL from the white paper [1]. The long distance and heavier quark contributions are under good control. The axial-vectors and the short-distance part provide the bulk of the error. Their errors were added linearly in [1] and include a guesstimate of the overlap between the short-distance from the quark-loop and the other contributions, including from other resonances above $1 \mathrm{GeV}$. The work described here [15-17] will allow to reduce the error.

Short-distance constraints can be used in many ways, we discuss those for the underlying object in HLbL, the derivative at $q_{4}=0$ of the four-point function of four electromagnetic currents:

$$
\Pi^{\mu \nu \lambda \sigma}=-i \int d^{4} x d^{4} y d^{4} z e^{-i\left(q_{1} \cdot x+q_{2} \cdot y+q_{3} \cdot z\right)}\left\langle T\left(j^{\mu}(x) j^{\nu}(y) j^{\lambda}(z) j^{\sigma}(0)\right)\right\rangle
$$

In the notation of [14] the contribution to $a_{\mu}^{\mathrm{HLbL}}$ comes via 


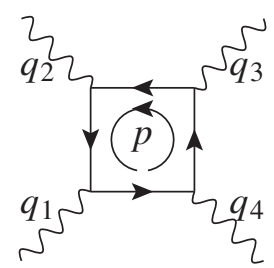

(a)

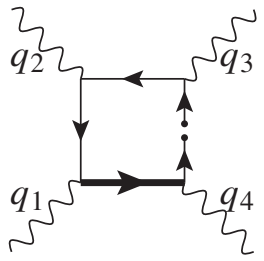

(b)

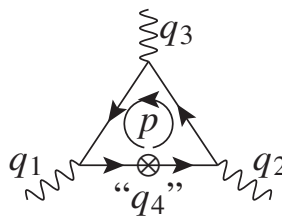

(c)

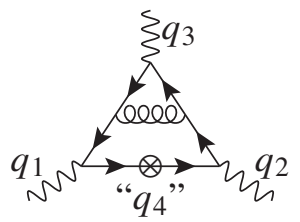

(d)

Figure 2: (a) The (constituent) quark-loop. (b) The quark-loop with an insertion of the quark-antiquark vacuum-expectation-value. (c) An insertion of the back-ground field. (d) An example of a gluonic correction.

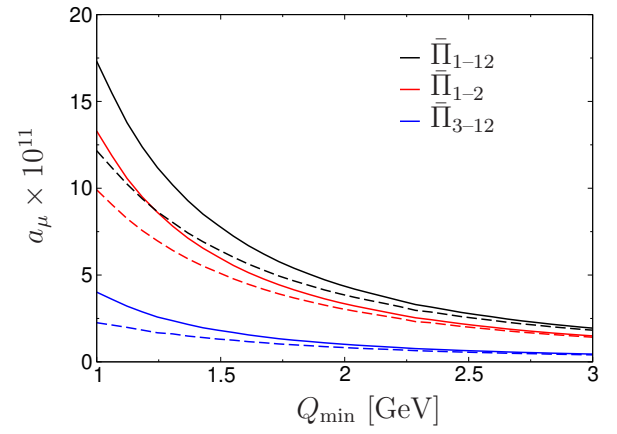

(a)

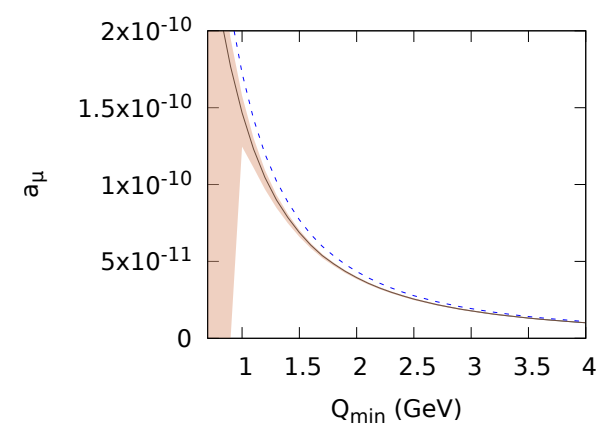

(b)

Figure 3: The contribution with $Q_{i} \geq Q_{\min }$ for (a) $M_{Q}=0$ and $M_{Q}=0.3 \mathrm{GeV}$, from [1]; (b) the massless quark-loop (LO) and the gluonic correction (NLO) and its uncertainty due to varying $\alpha_{S}$, from [17].

$$
\begin{aligned}
\Pi^{\mu \nu \lambda \sigma} & =\sum_{i=1}^{54} T_{i}^{\mu \nu \lambda \sigma} \hat{\Pi}_{i},\left.\quad \frac{\delta \Pi^{\mu \nu \lambda \sigma}}{\delta q_{4 \rho}}\right|_{q_{4}=0}=\left.\sum_{i=1}^{54} \frac{\delta T_{i}^{\mu \nu \lambda \sigma}}{\delta q_{4 \rho}} \hat{\Pi}_{i}\right|_{q_{4}=0}, \quad Q_{3}^{2}=Q_{1}^{2}+Q_{2}^{2}+2 Q_{1} Q_{2} \tau, \\
a_{\mu}^{\mathrm{HLbL}} & =\frac{2 \alpha^{3}}{3 \pi^{2}} \int_{0}^{\infty} d Q_{1} d Q_{2} Q_{1}^{3} Q_{2}^{3} \int_{-1}^{1} d \tau \sqrt{1-\tau^{2}} \sum_{i=1}^{12} \hat{T}_{i}\left(Q_{1}, Q_{2}, \tau\right) \bar{\Pi}_{i}\left(Q_{1}, Q_{2}, \tau\right) .
\end{aligned}
$$

The $12 \bar{\Pi}_{i}$ can be obtained from the $\hat{\Pi}_{i}$ for $i=1,4,7,17,39,54$. We use $Q_{i} \cdot Q_{j}=-q_{i} \cdot q_{j}$.

\section{Constituent quark-loop and first attempt: naive OPE}

The constituent quark-loop was used for full HLbL estimates since the 1970s and was often recalculated. It was used in [8] for a simple match with short-distances. The total contribution with $M_{Q}=0.3 \mathrm{GeV}$ is $a_{\mu}^{\mathrm{HLbLQ}}=54 \cdot 10^{-11}$ and above $1 \mathrm{GeV}$ only $12 \cdot 10^{-11}$. Massless above $1 \mathrm{GeV}$ is $17 \cdot 10^{-11}$. One result is that the latter is the first term in a well defined QCD operator-productexpansion (OPE). The $Q_{\mathrm{min}}$-dependence is shown in Fig. 3(a) and is $1 / Q_{\min }^{2}$ as expected.

The usual OPE applied to (1) puts all currents close together or assumes that all $\left|Q_{i} \cdot Q_{j}\right|$ are large. We then take the derivative w.r.t. $q_{4}$ and send $q_{4} \rightarrow 0$. The simple quark-loop of Fig. 2(a) is well defined, with $Q_{1}^{2}, Q_{2}^{2}, Q_{3}^{2} \geq Q_{\mathrm{min}}^{2} \gg \Lambda_{\mathrm{QCD}}^{2}$. The loop integration over $p$ damps the infrared (IR) divergence. The problem comes when we try to add higher orders in the OPE, e.g. a vacuum expection value contribution shown in Fig. 2(b). The thick propagator becomes divergent when we send $q_{4} \rightarrow 0$. This method cannot be used to obtain a proper OPE for the HLbL contribution to $a_{\mu}$.

\section{OPE in a background field}

The same problem with limits appeared in the QCD sum rule calculations for electromagnetic radii and magnetic moments $[18,19]$, solved by doing the OPE in a background field. We treat 


\begin{tabular}{|l|l|r|r|}
\hline Order & Contribution & $\begin{array}{r}Q_{\min }= \\
1 \mathrm{GeV}\end{array}$ & $\begin{array}{r}Q_{\min }= \\
2 \mathrm{GeV}\end{array}$ \\
\hline $1 / Q_{\min }^{2}$ & quark-loop & $1.73 \cdot 10^{-10}$ & $4.35 \cdot 10^{-11}$ \\
\hline \multirow{2}{*}{$1 / Q_{\min }^{4}$} & quark-loop, & $-5.7 \cdot 10^{-14}$ & $-3.6 \cdot 10^{-15}$ \\
& $m_{q}^{2}$ & $-1.2 \cdot 10^{-12}$ & $-7.3 \cdot 10^{-14}$ \\
\hline $1 / Q_{\min }^{6}$ & $X_{2, m}$ & $6.4 \cdot 10^{-15}$ & $1.0 \cdot 10^{-16}$ \\
& $X_{2, m^{3}}$ & $-3.0 \cdot 10^{-14}$ & $-4.7 \cdot 10^{-16}$ \\
& $X_{3}$ & $3.3 \cdot 10^{-14}$ & $5.3 \cdot 10^{-16}$ \\
& $X_{4}$ & $-1.8 \cdot 10^{-13}$ & $-2.8 \cdot 10^{-15}$ \\
& $X_{5}$ & $1.3 \cdot 10^{-13}$ & $2.0 \cdot 10^{-15}$ \\
& $X_{6}$ & $9.2 \cdot 10^{-13}$ & $1.5 \cdot 10^{-14}$ \\
& $X_{7}$ & $3.0 \cdot 10^{-13}$ & $4.7 \cdot 10^{-15}$ \\
& $X_{8,1}$ & $-1.3 \cdot 10^{-13}$ & $-2.0 \cdot 10^{-15}$ \\
& $X_{8,2}$ &
\end{tabular}

(a)

\begin{tabular}{|l|r|r|}
\hline & $\begin{array}{r}\text { Quark- } \\
\text { loop }\end{array}$ & $\begin{array}{r}\text { Gluon corrections } \\
\left(\frac{\alpha_{s}}{\pi} \text { units }\right)\end{array}$ \\
\hline $\bar{\Pi}_{1}$ & 0.0084 & -0.0077 \\
\hline $\bar{\Pi}_{2}$ & 13.28 & -12.30 \\
\hline $\bar{\Pi}_{3}$ & 0.78 & -0.87 \\
\hline $\bar{\Pi}_{4}$ & -2.25 & 0.62 \\
\hline $\bar{\Pi}_{5}$ & 0.00 & 0.20 \\
\hline $\bar{\Pi}_{6}$ & 2.34 & -1.43 \\
\hline $\bar{\Pi}_{7}$ & -0.097 & 0.056 \\
\hline $\bar{\Pi}_{8}$ & 0.035 & 0.41 \\
\hline $\bar{\Pi}_{9}$ & 0.623 & -0.87 \\
\hline $\bar{\Pi}_{10}$ & 1.72 & -1.61 \\
\hline $\bar{\Pi}_{11}$ & 0.696 & -1.04 \\
\hline $\bar{\Pi}_{12}$ & 0.165 & -0.16 \\
\hline Total & 17.3 & -17.0 \\
\hline \multicolumn{2}{|c}{ (b) } \\
\hline
\end{tabular}

Table 2: (a) Numerical results for the massless quark-loop and the contributions from condensates. Table from [16]. (b) Numerical results for the twelve different $\bar{\Pi}_{i}$ contributions from the quark-loop and the gluonic corrections in units of $10^{-11}$ and $10^{-11} \alpha_{S} / \pi$. Table from [17].

the $q_{4}$-leg as a constant background field and do the OPE with this. IR divergences are absorbed in condensates and the expansion where the three other currents are close, $Q_{1}^{2}, Q_{2}^{2}, Q_{3}^{2} \gg \Lambda_{\mathrm{QCD}}^{2}$, can be done. A new condensate is the magnetic quark-susceptibility $X_{q},\left\langle\bar{q} \sigma_{\alpha \beta} q\right\rangle \equiv e_{q} F_{\alpha \beta} X_{q}$. Pictorially one replaces Fig. 2(b) with Fig. 2(c). The IR divergence in Fig. 2(b) is absorbed via $X_{q}$

The first term in this expansion is the massless quark-loop [15], the next term is proportional to the quark-mass and the magnetic susceptibility. More details, including the detailed treatment of the IR divergences, and the expansion including the first three orders are in [16]. As an example of the IR interplay, the $m_{q}^{2}$ corrections to the quark-loop mix with the magnetic susceptibility contribution.

Using phenomenological estimates or lattice calculations, values for all condensates were obtained in [16]. The contribution to $a_{\mu}$ is shown in Tab. 2. The main message is that all higher orders are small because of small quark-masses and condensates for a lower cut-off above $1 \mathrm{GeV}$.

\section{Perturbative corrections and Melnikov-Vainshtein limit}

Since the nonperturbative corrections were small, the last place where large corrections might exist are the perturbative corrections, i.e. gluonic corrections to the massless quark-loop as in Fig. 2(d). We used the method of master integrals, all integrals are known analytically [20], and dimensional regularization. IR and UV divergences cancel. Results and more details are in [17]. Numerical instabilities appear in the expressions near $\lambda=Q_{1}^{4}+Q_{2}^{4}+Q_{3}^{4}-2 Q_{1}^{2} Q_{2}^{2}-2 Q_{2}^{2} Q_{3}^{2}-2 Q_{3}^{2} Q_{1}^{2}=$ 0 but one can expand analytically to solve this. The full expressions and all expansions are in the supplementary material of [17]. Numerical results are shown in Tab. 2(b). Corrections are typically of order $-10 \%$. The main uncertainty, the band in Fig. 3(b), is the value of $\alpha_{S}$.

A constraint was derived in [21] for two of the $q_{i}^{2}$ much larger than the third. Consequences are very strong because of the anomaly. Recent discussions are in [22-28]. In particular, the massless quark-loop reproduces this constraint [17, 25] as argued earlier [29]. The short-distance gluonic 
corrections to this constraint, see [23], is correctly reproduced by our gluonic corrections.

\section{Conclusions}

We studied the short-distance contributions for HLbL for the muon $g-2$. We have shown that this can be done properly in QCD and found that there are no unusually large corrections.

\section{Acknowledgments}

We thank Laetitia Laub for a fruitful and enjoyable collaboration. This research is supported in part by the Albert Einstein Center for Fundamental Physics at Universität Bern (NHT), the Swedish Research Council grants contract numbers 2016-05996 and 2019-03779 (JB) and the Agence Nationale de la Recherche (ANR) under grant ANR-19-CE31-0012 (project MORA) (ARS).

\section{References}

[1] T. Aoyama et al., Phys. Rept. 887 (2020) 1 [2006. 04822].

[2] Muon g-2 collaboration, Phys. Rev. D73 (2006) 072003 [hep-ex/0602035].

[3] Muon G-2 collaboration, Phys. Rev. Lett. 126 (2021) 141801 [2104.03281].

[4] M. Fertl et al., The muon $g$ - 2 experiment at FNAL, PoS (PANIC2021) 014.

[5] E.-H. Chao et al., Eur. Phys. J. C 81 (2021) 651 [2104.02632].

[6] T. Blum et al., Phys. Rev. Lett. 124 (2020) 132002 [1911.08123].

[7] J. Bijnens, E. Pallante and J. Prades, Phys. Rev. Lett. 75 (1995) 1447 [hep-ph/9505251].

[8] J. Bijnens, E. Pallante and J. Prades, Nucl. Phys. B 474 (1996) 379 [hep-ph/9511388].

[9] M. Hayakawa and T. Kinoshita, Phys. Rev. D 57 (1998) 465 [hep-ph/9708227].

[10] J. Bijnens, E. Pallante and J. Prades, Nucl. Phys. B 626 (2002) 410 [hep-ph/0112255].

[11] M. Hayakawa and T. Kinoshita, hep-ph/0112102.

[12] J. Prades et al., Adv. Ser. Direct. High Energy Phys. 20 (2009) 303 [0901.0306].

[13] G. Colangelo, M. Hoferichter, M. Procura and P. Stoffer, JHEP 09 (2015) 074 [1506 . 01386].

[14] G. Colangelo, M. Hoferichter, M. Procura and P. Stoffer, JHEP 04 (2017) 161 [1702 . 07347].

[15] J. Bijnens et al., Phys. Lett. B798 (2019) 134994 [1908.03331].

[16] J. Bijnens et al., JHEP 10 (2020) 203 [2008 . 13487].

[17] J. Bijnens et al., JHEP 04 (2021) 240 [2101.09169].

[18] I.I. Balitsky and A.V. Yung, Phys. Lett. 129B (1983) 328.

[19] B.L. Ioffe and A.V. Smilga, Nucl. Phys. B232 (1984) 109.

[20] F. Chavez and C. Duhr, JHEP 11 (2012) 114 [1209.2722].

[21] K. Melnikov and A. Vainshtein, Phys. Rev. D70 (2004) 113006 [hep-ph/0312226].

[22] M. Knecht, JHEP 08 (2020) 056 [2005 . 09929].

[23] J. Lüdtke and M. Procura, Eur. Phys. J. C 80 (2020) 1108 [2006. 00007].

[24] P. Masjuan, P. Roig and P. Sanchez-Puertas, 2005.11761.

[25] G. Colangelo et al., Phys. Rev. D 101 (2020) 051501 [1910.11881].

[26] G. Colangelo et al., Eur. Phys. J. C 81 (2021) 702 [2106.13222].

[27] J. Leutgeb and A. Rebhan, Phys. Rev. D 101 (2020) 114015 [1912.01596].

[28] L. Cappiello et al., Phys. Rev. D 102 (2020) 016009 [1912 . 02779].

[29] J. Bijnens and J. Prades, Mod. Phys. Lett. A 22 (2007) 767 [hep-ph/0702170]. 